\title{
Pancreatic cystosis: A rare pancreatic manifestation in cystic fibrosis
}

\author{
Mohammad A Wazzan \\ Radiology Department, The First Clinic, Jeddah, Saudi Arabia. \\ Correspondence to: Mohammad A Wazzan, E-mail: mohammad.wazzan@gmail.com \\ Received December 3, 2014. Accepted December 9, 2014
}

\begin{abstract}
Cystic fibrosis (CF) is a multisystemic, autosomal recessive disorder that predominantly affects infants, children, and young adults. Manifestations of CF involve the lung and gastrointestinal tract, including the pancreas. Pancreatic cystosis is a rare condition typically seen in CF during the second decade of life. It has characteristic magnetic resonance imaging (MRI) and ultrasound (US) features. A large cystic mass replacing the pancreas has been found by US and MRI study carried out as a part of clinical workup for a 17-year-old girl, known to have CF and presented with epigastric pain and a palpable mass. There was no history of trauma or clinical evidence of pancreatitis. On the basis of MRI findings and the patient age, a radiological diagnosis of pancreatic cystosis is securely made. Biopsy should be preserved for those who have atypical presentation.
\end{abstract}

KEY WORDS: Pancreatic cystosis, cystic fibrosis, pancreatic cystic mass, pediatric

\section{Introduction}

Pancreatic cystosis is extremely rare, and to our knowledge, only a few cases have been reported in the literature. ${ }^{[1]}$ It is typically seen in the second decade of life and described as complete replacement of the pancreas by cysts of different sizes, ranging from 1 to $12 \mathrm{~cm}$, and lack of normal pancreatic tissue identification. ${ }^{[2]}$

\section{Case Report}

A 17-year-old girl, known to have cystic fibrosis, presented to the emergency department with recurrent epigastric pain and a palpable mass. The pain was diffuse and continuous, without exacerbating or relieving factors. Medical history was significant for cystic fibrosis, pancreatic insufficiency, and pulmonary disease. Surgical history showed no abdominal surgeries. Medications included pancreatic enzyme supplements, proton-pump inhibitors, $\beta$-agonist, fat-soluble vitamin supplements, dornase alpha, and inhaled tobramycin. Physical examination showed diffuse abdominal tenderness to light

\begin{tabular}{|l|l|}
\hline Website: http://www.jmsph.com & Access this article online \\
\hline DOl: 10.5455/ijmsph.2015.0312201446 & \\
\hline
\end{tabular}

palpation and a large left hypochondrial mass. Pulmonary examination showed poor respiratory effort but clear breath sounds. There was no clinical or biochemical evidence of pancreatitis. A bedside ultrasound was performed [Figure 1], which showed a large septated cystic mass occupying the expected location of the pancreas. There was no solid component or significant vascularity. The pancreatic duct was not clearly visualized. The liver, common bile duct, spleen, and the kidneys were normal. The CT scan was withheld to avoid radiation.

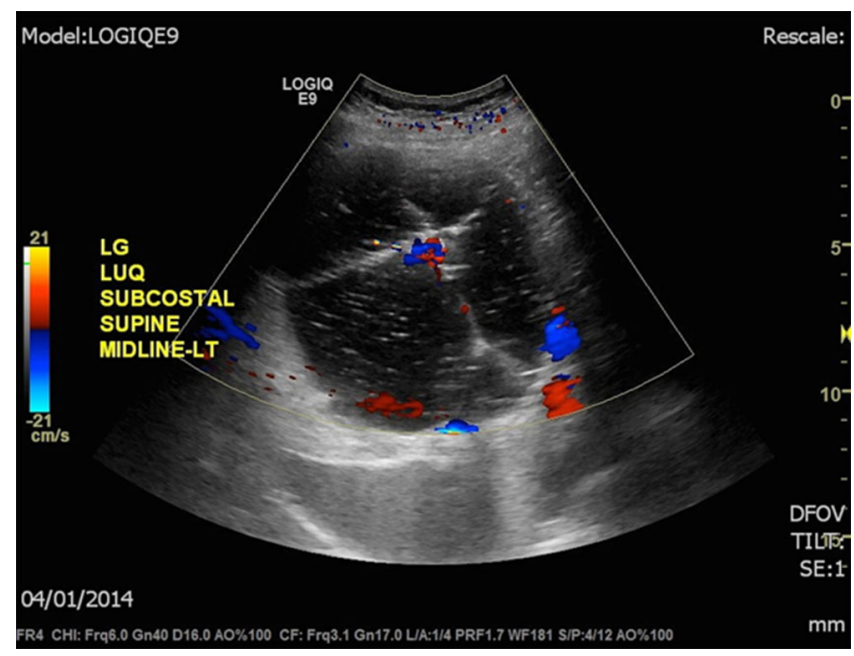

Figure 1: Doppler ultrasound of the abdomen shows multiseptated cystic mass with internal echogenicity. There is mild vascularity in the septations. 


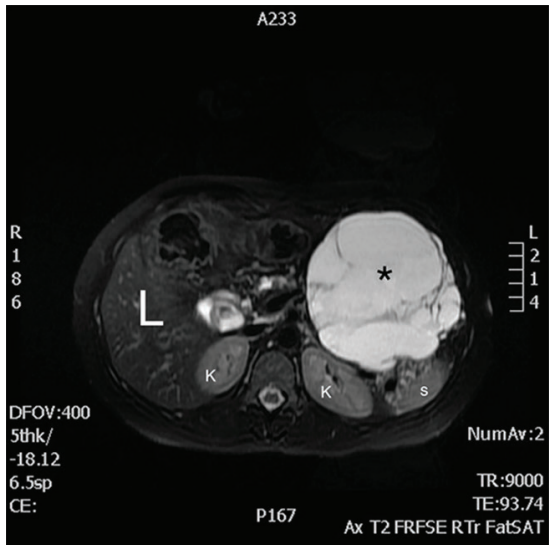

(a)

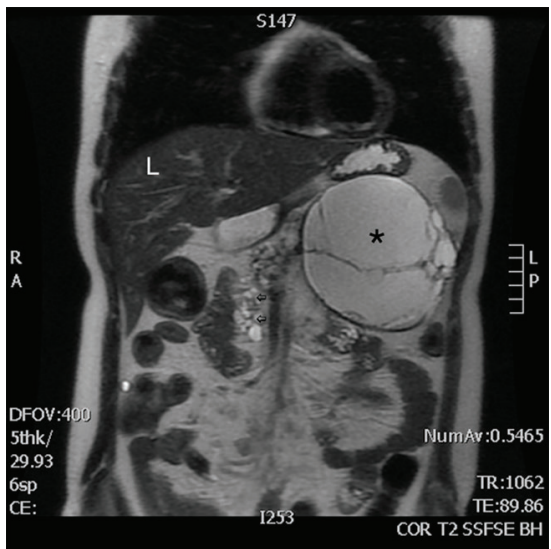

(c)
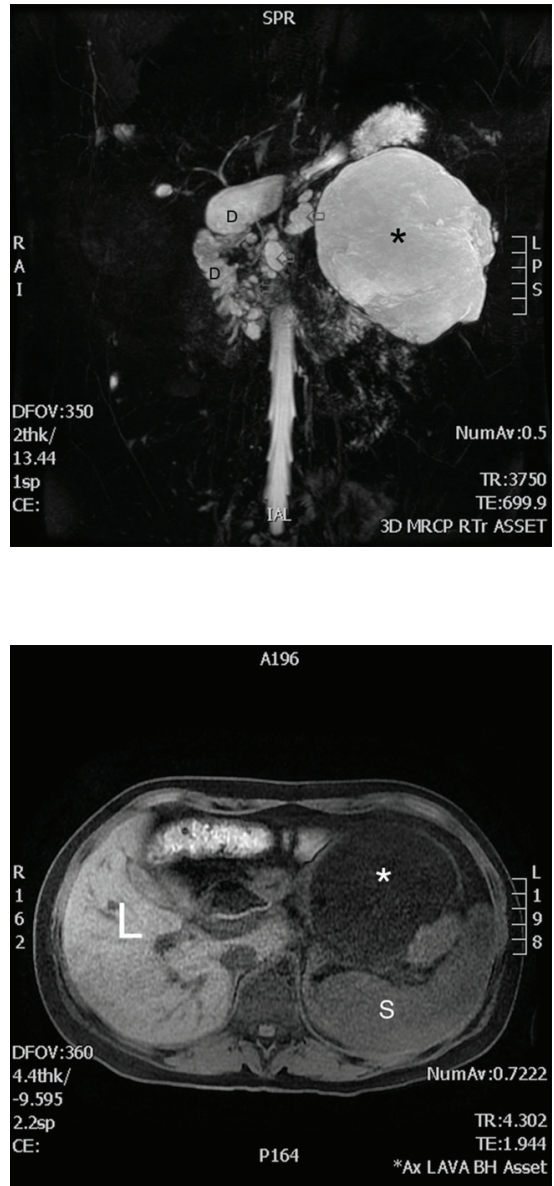

(d)

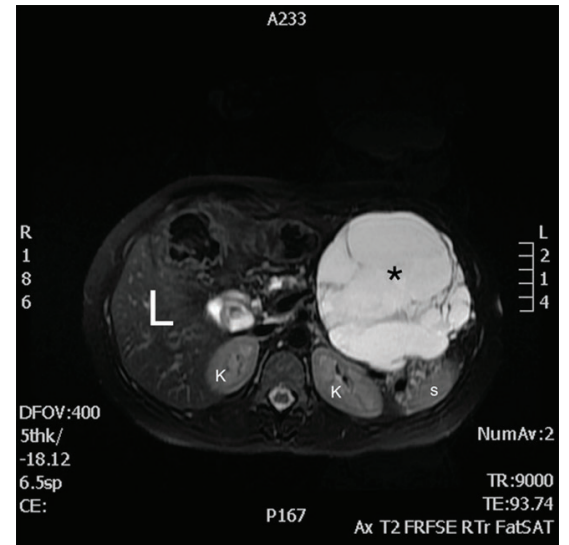

(b)

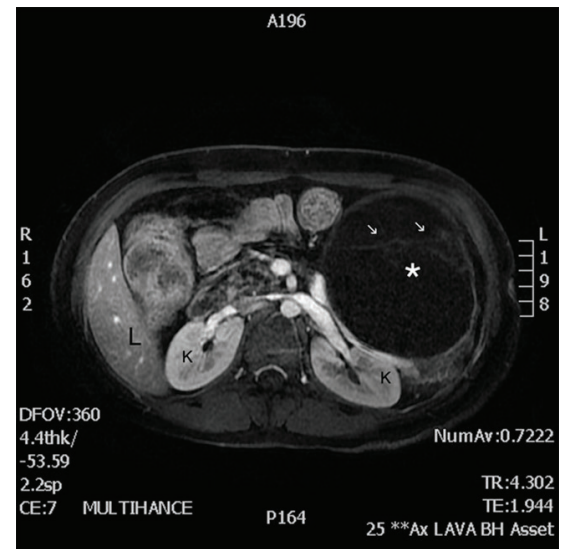

(e)

Figure 2: An abdominal MRI. (a) Axial T2 fat saturated obtained at the level of the pancreases and (b) coronal T2 shows a large multiseptated hyperintense cystic mass $(*)$ in the left hypochondrium. (c) Magnetic resonance cholangiopancreatography (MRCP) shows multiple smaller cysts (empty arrows) replacing the body and the head of the pancreas. (d) Axial T1 fat saturated and (e) enhanced axial T1 show homogenous hypointensity of the mass $(*)$ and enhancement of the septations (solid arrows). The liver (L), kidneys (K), spleen (S), and the duodenum (D) are normal.

An abdominal magnetic resonance imaging (MRI) [Figure 2] showed multiple innumerable cysts, ranging from 1 to $18 \mathrm{~cm}$, replacing the pancreas. Most of the cysts were hypointense in $\mathrm{T} 1$ sequence, and almost all of them were T2 hyperintense. Some of the cysts were T1 hyperintense, considered as high protein content. There were multiple fluid/ fluid levels, likely related to prior hemorrhagic episodes. There were a few enhancing septations and no solid component. No normal pancreatic tissue was appreciated. A radiological diagnosis of pancreatic cystosis was performed based on the typical MRI and ultrasound features.

\section{Discussion}

Cystic fibrosis is the most lethal autosomal recessive disease among Caucasian people, with approximately incidence of $1 / 2500$ live birth. ${ }^{[1,3]}$ The pancreas is involved in approximately $85 \%$ of patients with cystic fibrosis at some time in their lives. ${ }^{[4]}$ The clinical symptoms, including steatorrhea, fat intolerance, abdominal pain, and failure to thrive, will be shown after $90 \%$ loss of pancreatic tissue.

Four imaging manifestation of the pancreatic involvement have been discussed in the literature ${ }^{[1]}$ : (1) partial fibro-fatty replacement of the pancreatic tissue, which is most common in the tail as compared to the body; (2) complete fibro-fatty replacement; (3) atrophy without fibro-fatty replacement; and (4) pancreatic cystosis.

The partial and complete fibro-fatty replacement, and the atrophy represent $16 \%, 42 \%$, and $24 \%$ of the cases, respectively. ${ }^{[5]}$ They can be associated with micro- or macrocysts.

Pancreatic cystosis is extremely rare. It is typically seen in the second decade of life and described as complete replacement of the pancreas by cysts of different sizes, ranging from 1 to $12 \mathrm{~cm}$, and lack of normal pancreatic tissue identification. The main etiology has been thought to be 
related to a defect in bicarbonate transport, ${ }^{[6]}$ which will lead to diluted protein and subsequent ductal ectasia.

The top differential diagnosis for pancreatic cystic lesion would include the following:

- Pancreatic psudocyst, which usually is less numerous and associated with inflammatory process or trauma. ${ }^{[7]}$

- Mucinous and serous cystic diseases that typically involve middle-aged and older women.

- Intraductal papillary mucinous tumor typically shows communication with the pancreatic duct.

- Pancreatic involvement in polycystic kidneys disease, which can be excluded if the kidneys are normal.

- Von Hippel-Lindau disease, which is also excluded in the sitting of normal kidneys.

On the basis of MRI findings and the patient age, those differential diagnoses were excluded and a diagnosis of pancreatic cystosis was made. Biopsy has been deemed unnecessary in patent with pancreatic cystosis. ${ }^{[1]}$

\section{Conclusion}

Conclusively, in case of macrocystic replacement of the pancreas seen in a patient with cystic fibrosis, an imagingbased diagnosis of pancreatic cystosis should be made.

\section{References}

1. van Rijn RR, Schilte PP, Wiarda BM, Taminiau JA, Stoker J. Case 113: pancreatic cystosis. Radiology 2007;243:598-602.

2. Berrocal T, Pajares MP, Zubillaga AF. Pancreatic cystosis in children and young adults with cystic fibrosis: sonographic, CT, and MRI findings. AJR Am J Roentgenol 2005;184(4):1305-9.

3. Hernanz-Schulman M, Teele R, Perez-Atayde A, Zollars L, Levine $\mathrm{J}$, Black $\mathrm{P}$, et al. Pancreatic cystosis in cystic fibrosis. Radiology 1986;158:629-31.

4. Nousia-Arvanitakis S. Cystic fibrosis and the pancreas: recent scientific advances. J Clin Gastroenterol 1999;29:138-42.

5. Feigelson J, Pécau $Y$, Poquet $M$, Terdjman $P$, Carrère $J$, Chazalette $\mathrm{J}$, et al. Imaging changes in the pancreas in cysticfibrosis: aretrospective evaluation of 55 cases seen overa period of 9 years. J Pediatr Gastroenterol Nutr 2000;30(2): 145-51.

6. Taylor C, Aswani N. The pancreas in cystic fibrosis. Paediatr Respir Rev 2002;3(1):77-81.

7. Fulcher AS, Turner MA. MR pancreatography: a useful tool for evaluating pancreatic disorders. Radiographics 1999;19(1): $5-24$.

How to cite this article: Wazzan MA. Pancreatic cystosis: A rare pancreatic manifestation in cystic fibrosis. Int $\mathrm{J}$ Med Sci Public Health 2015;4:307-309

Source of Support: Nil, Conflict of Interest: None declared. 